\title{
Neutron Radiography, Techniques and Applications
}

Domanus, J.C.

Publication date:

1987

Document Version

Publisher's PDF, also known as Version of record

Link back to DTU Orbit

Citation (APA):

Domanus, J. C. (1987). Neutron Radiography, Techniques and Applications. Risø National Laboratory. Risø-M No. 2672

\section{General rights}

Copyright and moral rights for the publications made accessible in the public portal are retained by the authors and/or other copyright owners and it is a condition of accessing publications that users recognise and abide by the legal requirements associated with these rights.

- Users may download and print one copy of any publication from the public portal for the purpose of private study or research.

- You may not further distribute the material or use it for any profit-making activity or commercial gain

- You may freely distribute the URL identifying the publication in the public portal

If you believe that this document breaches copyright please contact us providing details, and we will remove access to the work immediately and investigate your claim. 
RIS(
Teutron Radiography,
Techniques and Applications

J. C. Domanus

Risø National Laboratory, DK-4000 Roskilde, Denmark October 1987 
NEUYRON RADIOGRAPHY, TECHIQUES AND APPLICATIONS

J. C. Donanus

Abstract. After describing the principles of the "in pool" and "dry" installations, techniques used in neutron radiography are reviewed. Use of converter foils with silver halide films for the direct and transfer methods is described. Advantages of the use of nitrocellulose film for radiographying radioactive objacts are discussed. Dynamic imaging is shortly reviewed. Standardization in the field of neutron radiography (ASTM and Euratom Neutron Radiography Working Group) is described. The paper reviews main fields $o^{\circ}$ use of neutron radiography. Possibilities of use of neutron radiography at research reactors in various scientific, incistrial and other fields are mentioned. Examples are given of application of neutron radiography in industry and the nuclear field. 
This paper will be presented at the Seminar "Procedes de Control Non Destructifs" in Liége, Belgium (4 \& 5 February 1988) and at the Danish-Israeli Symposium on Non-Destructive Evaluation in Lyngby, Denmark (27 June - 1 July 1988).

ISBN $87-550-1371-6$

ISSN 0418-6435

Grafisk service, Risø, 1987 
1. PRINCIPLES OF NEUTRON RADIOGRAPHY 4

2. RADIATION SOURCES 6

3. NEUTRON RADIOGRAPHIC TECHNIQUES 6

3.1. Direct exposure technique 8

3.2. Transfer technique 9

3.3. Track-etch tecknique 10

4. VIEWING OF NEUTRON RADIOGRAPHS 12

5. DYNAMIC IMAGING 12

6. STANDARDIZATION 13

7. APPLICATIONS OF NEUTRON RADIOGRAPHY 14

7.1. Nuclear industry 16

Nuclear fuel 16

General $\quad 23$

7.2. Industrial applications 23

Explosives and pyrotechnical devices 23

$\begin{array}{ll}\text { Turbine blades } & 23\end{array}$

Aerospace $\quad 24$

Apollo

Ariane $\quad 24$

Corrosion $\quad 24$

Composite $\quad 25$

Other industrial applications $\quad 25$

Concrete $\quad 25$

Soil and rock 25

Electric contacts $\quad 25$

$\begin{array}{ll}\text { Electronic devices } & 26\end{array}$

Mechanical connectors and assemblies 26

Honeycomb $\quad 26$

Flow in heat pipes $\quad 26$

Metallurgy 26

Real-time $\quad 26$

Engine fluids 26

7.3. Non-industrial applications 27

Biology, medicine and dentistry 27

Histopathology 27

Dentistry 27

Biology and medicine $\quad 27$

Roots growing in soil $\quad 27$

Forensíc $\quad 27$

Arts $\quad 28$

$\begin{array}{ll}\text { Neutron autoradiography } & 28\end{array}$

$\begin{array}{ll}\text { ACKNOWLEDGEMENTS } & 28\end{array}$

$\begin{array}{lr}\text { REFERENCES } & 29\end{array}$ 


\section{PRINCIPLES OF NEUTRON RADIOGRAPHY}

All radiographic methods, whether making use of $X$-rays, gammarays or neutrons are based on the same general principle: that radiation is attenuated on passing through matter. The object under examination is placed in the incident radiation beam. After passing through, the beam that remains enters a detector that registers the fraction of the initial radiation intensity that has been attenuated, by each point in the object. Any inhomogeneity in the object or an internal defect (such as e.g. void, crack, porosity or inclusion) will show up as a change in radiation intensity reaching the detector.

Thus detection of defects in radiography is based on the observation of differences in radiation intensity after passing through the object under examination. This occurs according to the basic law of radiation attenuation:

$$
J=J_{0} \cdot e^{-\mu x}
$$

The radiation attenuation coefficient $\mu$ shows a continous curve for $x$-rays (over a wide range of wavelengths). This is not true, however, for neutrons and it happens that adjacent atomic number elements such as boron and carbon show for example marked differences in neutron attenuation. Because of this it is possible to detect hydrogen in zirconium. Conversly, dense materials such as lead, tungsten, or uranium are relatively easy to penetrate by neutrons.

A comparison of neutron and X-ray (125 kV) mass attenuation coefficients for various elements is given in fig. 1.

Another impotant advantage of neutron radiography is the possibllity of examining radioactive objects such as spent fuel elements directly. 


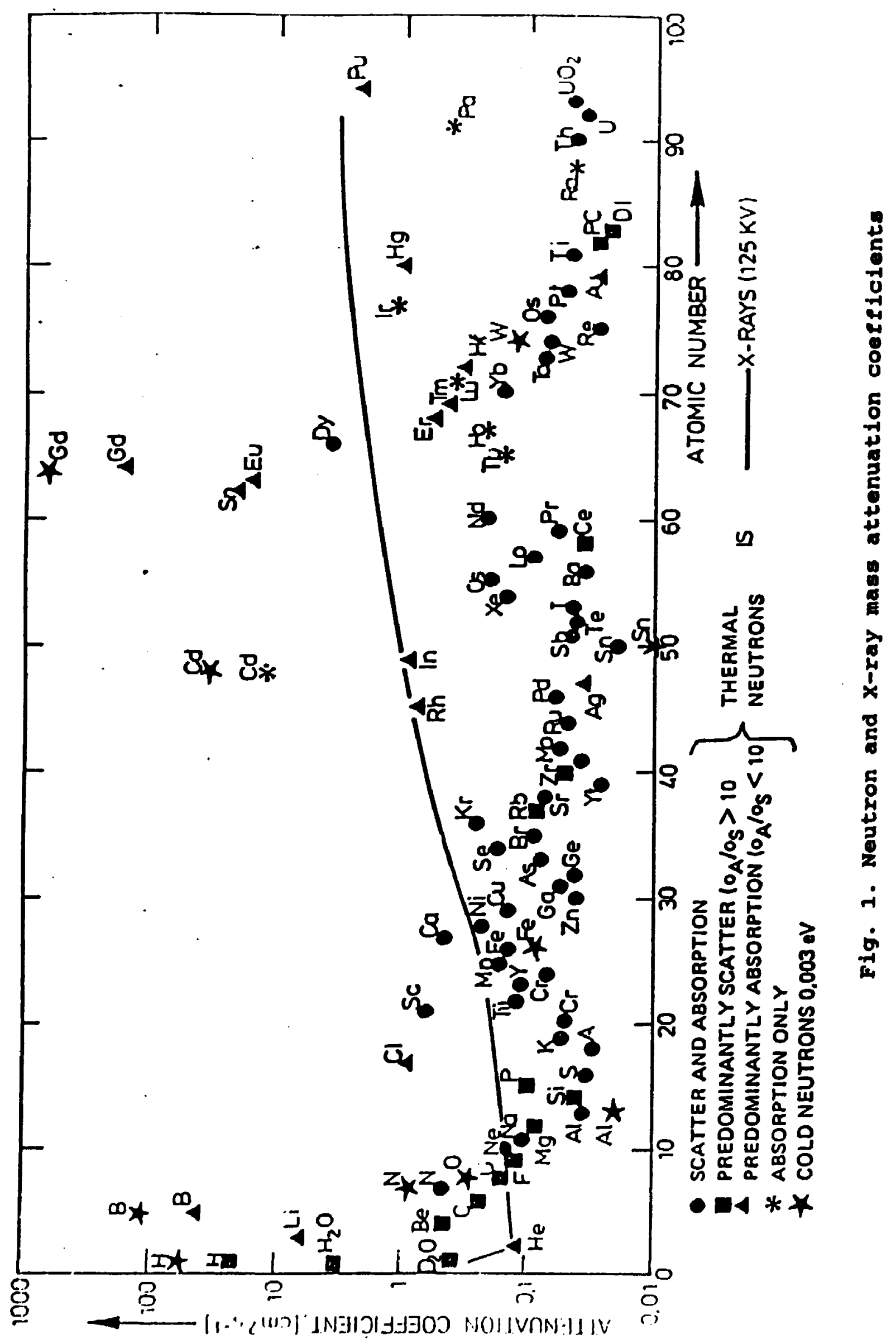




\section{RADIATION SOURCES}

There are three sources of neutrons available for neutron radiography: accelerators, radioisotopes and nuclear reactors. only the latter will be reviewed below. At present nuclear reactors provide the most intense neutron beams and therefore can produce neutron radiographs of the highest quality.

Two types of neutron radiographic facilities are used with nuclear reactors. In the "in pool" facility the whole neutron radiographic installation is immersed in the pool of the reactor. Here, irradiated reactor fuel rods, removed from the reactor core, are transferred to the neutron radirqraphic facility, where they are examined without removing then from the reactor pool. In the "dry" type facility a neutron beam taken out of the core of the reactoc is used outside the reactor for neutron radiography.

The "in pool" and "dry" neutron radiographic facilities are shown in fig. 2 .

\section{NEUTRON RADIOGRAPHIC TECHNIQUES}

$A B$ in $X$ - or gama-radiog:aphy, $x$-ray film is the medium for producing neutron radiographs. For radiographying radioactive materials the nitrocellulose film is also used.

Unfortunately, neutrons have very little direct effect on photographic film. Thus an intensifying screen of some kind is needed to improve the speed of the film. The nitrocellulose film must also be used with a converter screen, as neutrons do not directly affect this type of film.

of the many existing methods of recording neutron images, only those which are widely used in practice will be described here. They are the following: the direct and transfer technique using metal converter foils with $X$-ray $111 \mathrm{~m}$ and the track-etch technique using nitrocellulose film. The properties of metals used as converter foils for the direct and transfe techniques are 


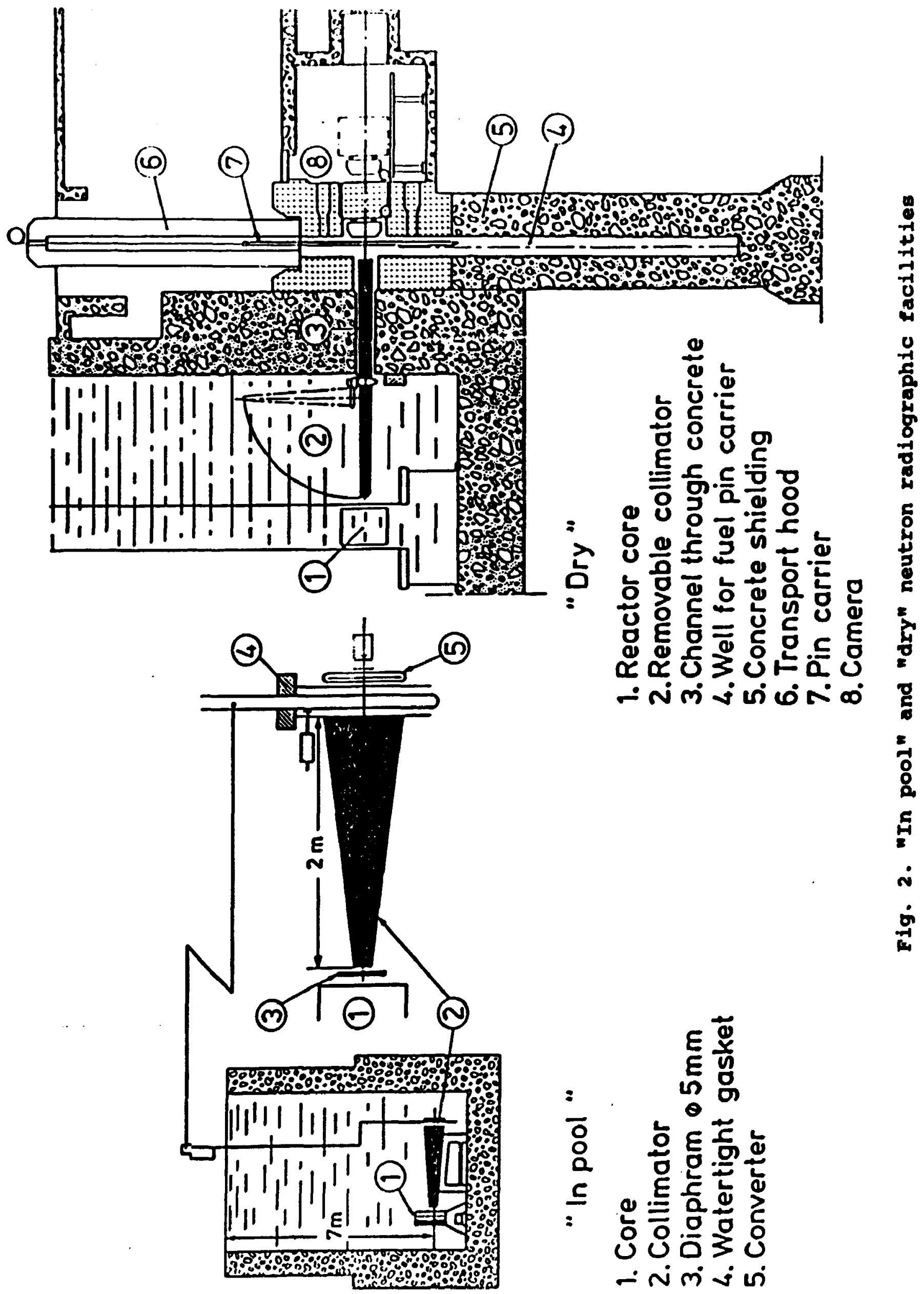


sumarized as follows:

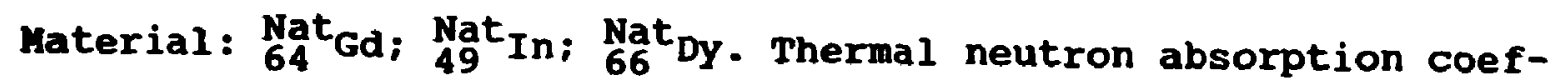
ficient: $140.3 ; 0.73 ; 3.01 \mathrm{~mm}^{-1}$. Predomiant nuclear reaction: $(n, r)$. Half-life of radiation emitter: promt; 54 min; $2.5 \mathrm{~h}$. Type and energy of radiation: IC $\beta^{-}, 71 \mathrm{keV}$ (main): $\beta^{-}, 1.28$ MeV $(\max ) ; \beta^{-}, 2.00 \mathrm{MeV}(\max )$.

Information about other techniques used in neutron radiography can be found in numerous references on the subject.

\subsection{Direct Exposure Technique}

In the direct exposure technuque a metal converter foil is placed in contact with $x$-ray film during the actual exposure (fig. 3). Usually, a single gadolinium back screen is used. This screen emits gamma-radiation on absorbing neutrons. The gammas in the spectrum from gadolinium are suitable for producing electrons by internal conversion. Those low-energy electrons essentially expose only the emulsion facing the gadolinium. Single coated slow $x$-ray films are therefore used with the direct technuque.

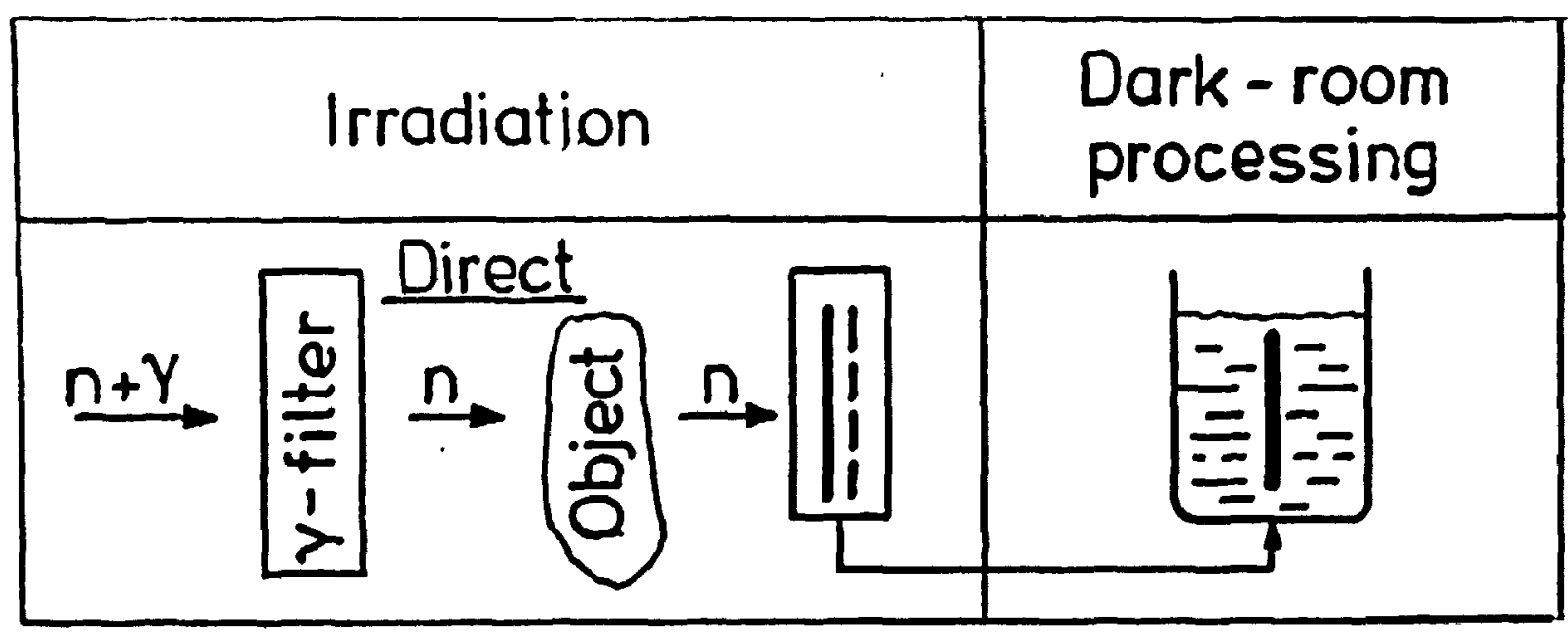

Fig. 3. Direct exposure technique 
At present a single, $25 \mu \mathrm{m}$ thick back screen is used. The screen is usually laminated to aluminium to facilitate handling. Vapour-phase deposited screens are also available, protected with a sapphire coating $1 \mu \mathrm{m}$ thick. To assure good contact between the film and screen vacuum cassettes are used.

With the direct exposure technique, using Ga converter foil, a very good spatial resolution can be achieved. A typical thermal neutron exposure for a slow, single coated $x$-ray film and a single $25 \mu \mathrm{m}$ Gd screen is about $10^{9} \mathrm{n} \cdot \mathrm{cm}^{-2}$.

\subsection{Transfer Technique}

In the transfer technique (fig. 4) only the converter screen is exposed directly to neutrons. The matal screen, placed in the neutron beam, becomes radioactive in proportion to the intensities in spatial neutron image. The screen is subsequently transferred from the neutron beam to a dark room where it is placed in close contact with the $x$-ray film using a vacuum cassette. The radioactive emission from the screen then produces an image on the film.

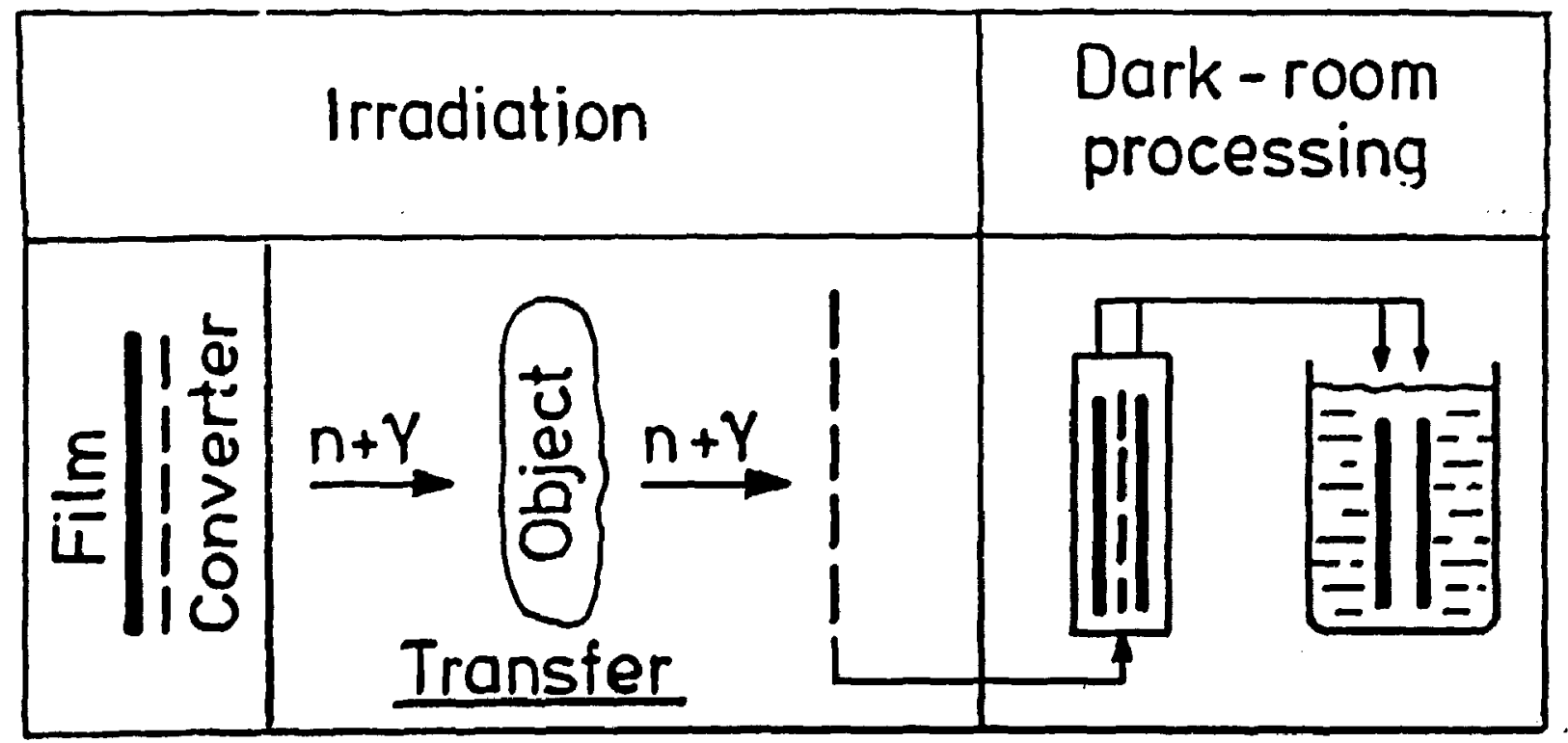

Fig. 4. Transfer technigue 
For the transfer texhnique a material must be chosen which is rapidly activated and has a rapid decay, so that it can be used again. Indium and dysprosium are the most commonly used materials.

The transfer method offers the advantage that the film, which is not present in the neutron beam, is not exposed to gammaradiation from a radioactive object or from gamma-rays in the neutron beam itself.

The more energetic $\beta$-particles emitted by the transfer screens have sufficient energy to penetrate normal photosensitive film completely. Thus both emulsions of a double coated X-ray film will contribute appreciably to the film density and to the unsharpness. To avoid the latter it is important to use single coated films.

For the transfer technique mainly indium and dysprosium foils are used. Dysprosium is a harder metal and therefore easier to handle and maintain. It also has a higher thermal cross section and longer half-life. Therefore, it can be used with weaker neutron beams.

For a $100 \mu \mathrm{m}$ Dy converter an exposure to neutrons of $3 \times 10^{9}$ n. $\mathrm{cm}^{-2}$, followed by an exposure of several half-lives of the converter to a medium speed $X$-ray film, will be needed. A good spatial resolution can be reached whit a single coated film.

\subsection{Track-etch Technique}

For neutron radiography of radioactive objects (such as irradiated nuclear fuel) nitrocellulose film is used as a neutron detector. This is a dielectric material which can detect charged particles by the radiation damage caused in it. Those charged particles are produced by an a-emitting converter screen. The radiation damage is made visible by etching in hot sonium hydroxide solution (e.g., in $108 \mathrm{NaOH}$ for $45 \mathrm{~min}$ at 50 c). The nitrocellulose film, sandwiched between two a-emitting converter screens, is placed directly in the neutron beam (fig. 
5) as it is insensitive to gamma-rays. As the nitrocellulose film is also insensitive to visible light the consecutive etching need not be done in a dark room.

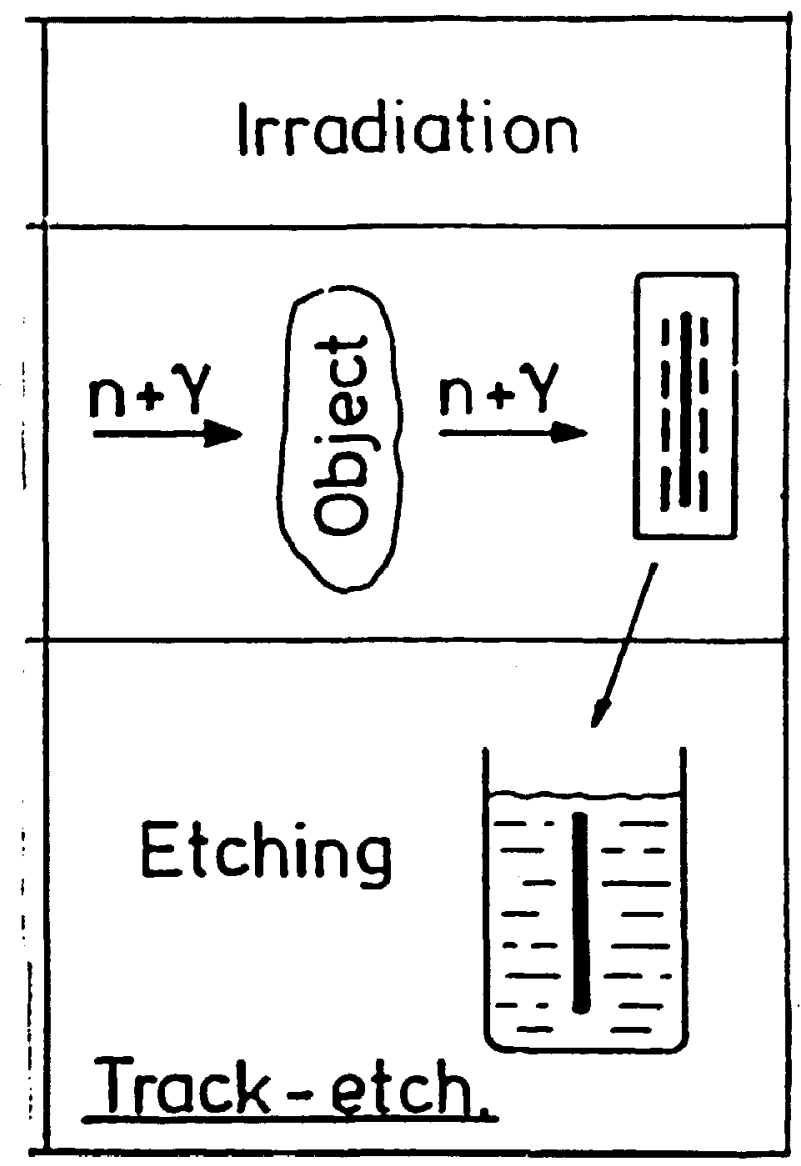

Fig. 5. Track-etch technique

Nitrocellulose film is available (from Kadak-Pathe, France) for neutron radiography in two film/converter variations. The first consists of a $100 \mu \mathrm{m}$ thick sheet of cellulose nitrate coated on both sides with lithium borate dispersed in a water-soluble binder, which acts as a converter screen by means of the $(n, a)$ reaction (CN 85 Type B). After irradiation the lithium borate coating is removed by washing and then film itself is etched.

The seconci variation consists of the same CN 85 nitrocellulose ilin (without coating) which is sandwiched between two converter screens (BN 1) made from natural boron, a $(n, a)$ converter. This converter is coated on a $100 \mu \mathrm{m}$ thick, very stable, polyester base and can be reused indefinitely. The efficeincy of the $B N 1$ is heigher than that of the CN 85 Type $B$ and there- 
fore requires exposure times only slightly longer than those for the transfer technique (with Dy converter and slow $x$-ray film). To establish perfect contact between the converter and nitrocellulose film the use of a vacuum cassette is essential.

When comparing nitrocellulose and $x$-ray films as midia for neutron radiography one can see that although the contrast on the nitrocellulose film is weaker the definition is better. A very good spatial resolution can be obtained. In comparison to the transfer technique there is no saturation of the converter; this can be advantageous when using low-intensity neutron sources. There is no handling of an active corverter, as in the transfer method. Finally, one shall mention the possibility of stopping the etching processing at intermediate stages. After evaluating the neutron radiograph at that stage the etching of the same nitrocellulose film can be further continued. Thus from a single exposure one can have several neutron radiographs of different densities and contrast.

\section{VIEWING OF NEUTRON RADIOGRAPHS}

Viewing neutron radiographs produced by the direct or transfer metod on $X$-ray film creates no special problems. Neutron radiographs produced by the track-etch technique on the other hand are unsuited for direct viewing because of their low contrast. The contrast can be significantly improved by printing the film on a high contrast film, using a point source enlarger. However, the nitrocellulose film can be directly examined by placing it between two polarizing filters.

\section{DYNAMIC IMAGING}

Although methods of dynamic imaging could more appropriately be called neutron fluoroscopy than neutron radiography, they will be shortly reviewed here. The principle of a TV system of neutron fluoroscopy is shown in fig. 6 . 


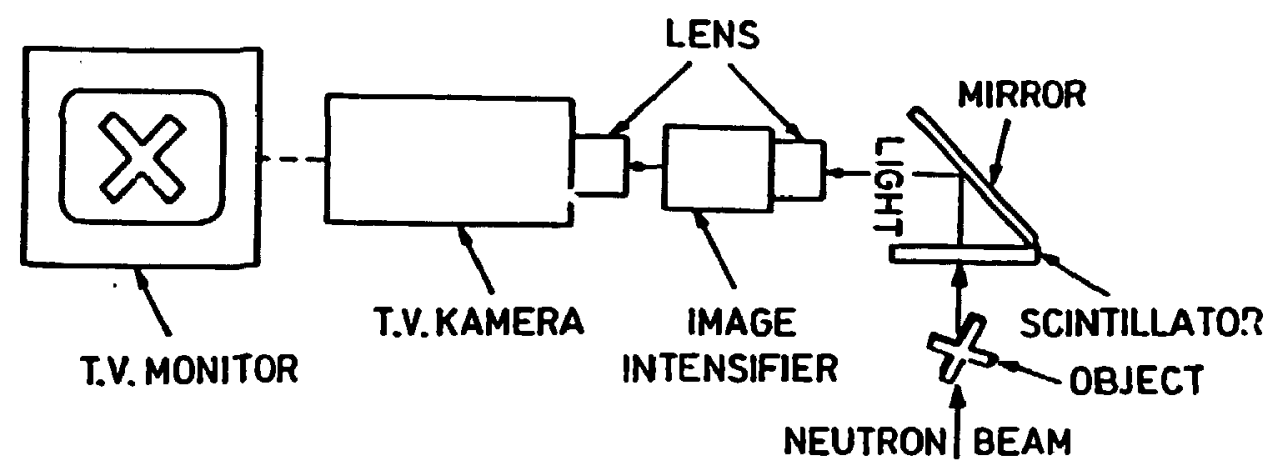

Fig. 6. TV neutron fluoroscopy

A more sophisticated system uses neutron image intensifier tubes with a front gadolinium screen. The secondary electrons from it are accelerated on to a scintillator screen on which the neutron picture can be observed. This neutron tube (or neutron camera) can be used with a TV system (camera and monitor) to provide a remote display and recording.

One of the most impress re applications of neutron fluoroscopy has been the cold neutron fluoroscopy of a running aircraft gas turbine engine or a car engine, in order to establish the dynamic distriburion of the lubricating oil through the oil passages within the engine.

\section{STANDARDIZATION}

As neutron radiography is a relatively new technique there are only few standards in that field. Besides the ASTM standardization the Euratom Neutron Radiography Working Group (NRWG) has started similar activity in 1979. As a result of this activity first a "Neutron Radiography Handbox" [1] and then "Reference Neutron Radiographs of Nuclear Reactor Fuel" [2] were pub11shed.

Under the NRWG test program beam purity and sensitivity indicators, together with a special calibration fuel pin were 
tested in 11 facilities of 7 centers in 6 countries. The purpose of this program is to choose the best image quality indicators for neutron radiography of nuclear fuel and to determine with which accuracy and by which methods dimensions from neutron radiographs can best be measured.

\section{APPLICATIONS OF NEUTRON RADIOGRAPHY}

Although neutron radiography is a well established non-destructive (NDT) testing technique ior quite a long time no special book on its applications has as yet been published. You cannot even find a comprehensive publication on the subject. For this reason the best source of information on applications of neutron radiography (NR) are the proceedings of the First (San Diego, 1981) and Second (Paris, 1986) World Conferences on Neutron Radiography $[3,4]$. While collecting detailed information about the design and construction of collimators for thermal neutron radiography, handbooks and general publications as well as conferences on NDT and NR were reviewed. They are listed in chapters 2 and 3 of [5], where adequate references can be found. They may also be useful for the search of information on applications of NR.

As early as 1965 Berger in the first book published on NR had written a separate chapter on its applications [6]. There he has suggested three main areas of applications for NR:

- reactor technology (fuel elements, shielding, coolants, inclusions):

- rocket and missile technology (fuels, components, gaskets) :

- general applications (biology, plastics, platings, diffusion, gaskets).

This general division is still valid today.

Many interesting examples of NR applications in France are given in [7]. 
A more detailed review of NR applications in the following fields can be found in [8]: nuclear, aerospace, explosives and ordnance as well as biology.

A list of reports which describe NR applications up to 1977 is given by Barton in [9].

A special issue of the IAEA Atomic Energy Review [10] deals also with the applications of NR for the control of nuclear fuel, studies of hydrogen transport, operations in industry with specific reference to metallurgy.

R. Matfield in [1] gives also a short review of NR applications, dividing them into nuclear, industrial, biomedical and other areas.

In the Nondestructive Testing Handbook of the ASNDT [11] Berger et al. [12] give a very short review of NR applications dividing them as follows:

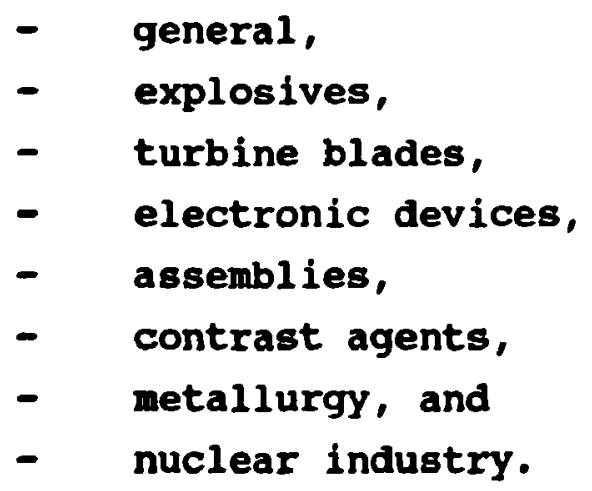

Based on the literature described above an overview of applications of NR will be given below.

As it is impossible to discuss in detail ali the various applications of NR, only a few examples were chosen, limited to those that could be illustrated with appropriate neutron radiographs. For those who would like to know about some more applications the review of literature on this subjects was given above. 
As is well known it is very difficult, or at times even impossible, to reproduce radiographs by ordinary printing methods. This is equally valid for neutron radiographs. Therefore in the printed version of this paper only very few neutron radiographs will be reproduced, whereas the oral presentation will make an ample use of them. This is possible because many slides of original neutron radiographs were kindly supplied by several authors of papers on applications of NR. Their contribution is kindly acknowledged herewith.

\subsection{Nuclear industry}

One of the main fields of application of NR is the nuclear industry. Here NR is applied mainly to control irradiated nuclear fuel as well as reactor control rods. Due to the high radioactivity of the nuclear fuel $x$-ray radiography cannot be used. Here the transfer and track-etch method of NR give the solution.

The defects can be located in different parts of the nuclear fuel. (The term "defect" is used to designate a change in appearance shown on an original radiograph of a particular part of the fuel as fabricated, to that shown on a subsequent radiograph, usually post irrisdiation).

Some typical examples of nuclear fuel pins are given in fig. 7 where all the components of the pins are listed. They represent pelletized, annual and vibro-compacted fuel. 

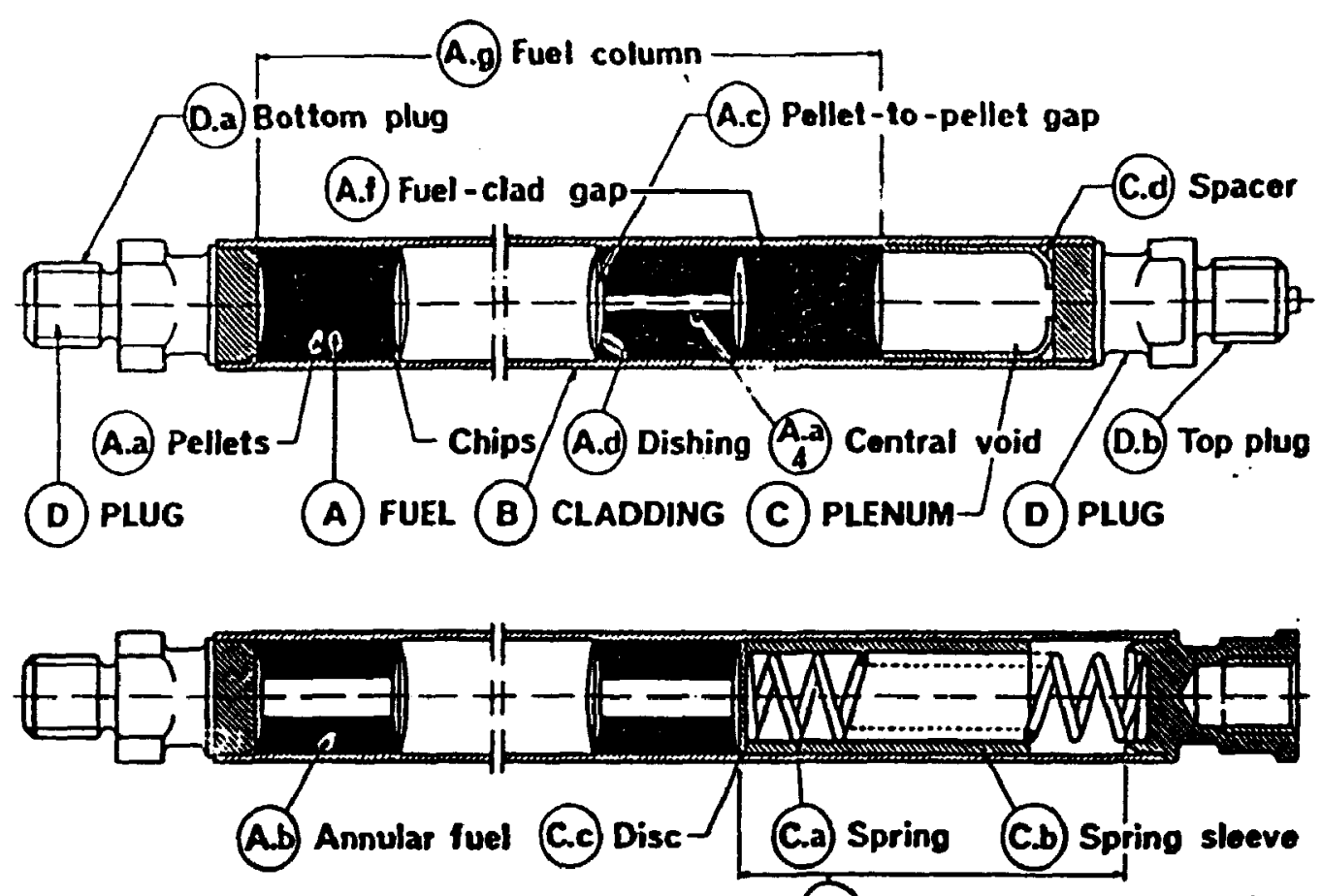

C. Top of fuel column to plug
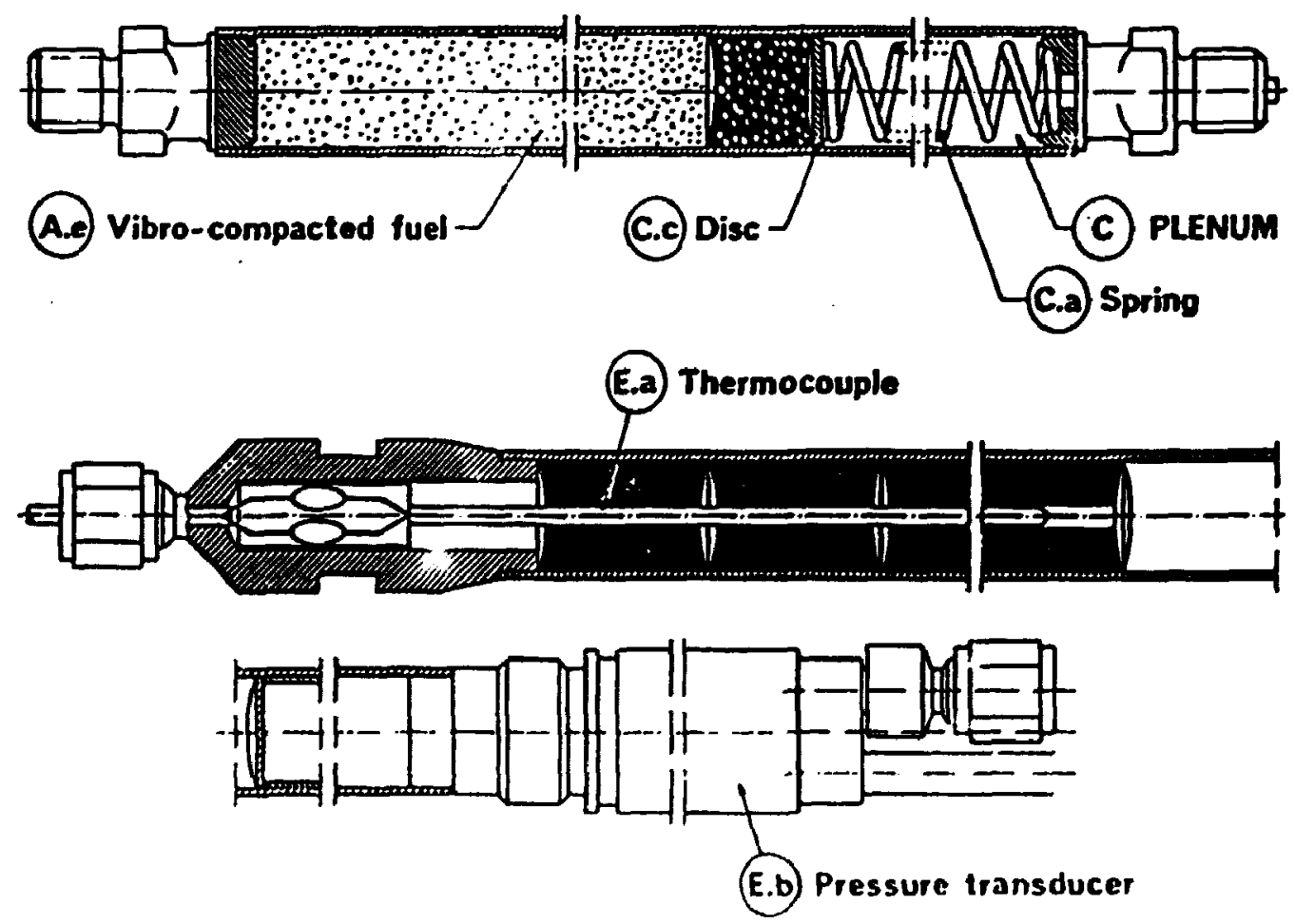

Fig. 7. Examples of nuclear fuel pins 
In other fields of industrial radiography defects that can be revealed by radiography have been classified and reference radiographs, showing typical defects (e.g. in weldings or castings) have been completed and published long ago. No such classification nor reference radiographs existed until recently in the field of neutron radiography.

The assessment of neutron radiographs of nuclear fuel elements can be much easier, faster and simpler if reference can be made to typical defects that can be revealed by neutron radiography. Therefore it was felt that a classification of such defects will help speed up the assessment procedure. Therefore such a classification was established by the NRWG, and "Reference Neutron Radiographs of Nuclear Reactor Fuel" were published in 1984 [2]. In this publication classification of neutron radiographic findings (in light water and fast reactor) is given together with 158 examples of defects in nuclear fuel as well as its different parts as fabricated. The text of this collection is produced both in English and French. Special terms used throughout the collection as well as some useful ones in the field of neutron radiography, are given in Danish, Dutch, Eng1ish, French, German and Italian.

From 158 neutron radiographic findings assembled in [2] some typical examples are given below (as enlargements of original radiographs on film).

On fig. 8 examples of pelletized fuel are given, whereas fig. 9 shows annular fuel, and fig. 10 vibro-compacted fuel. cladding is shown on fig. 11, plenum on fig. 12, plug on fig. 13 and instrumentation on fig. 14. As in the collection [2] the examples include also radiographs of nuclear fuel as fabricated (before irradiation). Thus it is easier to identify the changes that have occurred in the fuel during irradiation. 


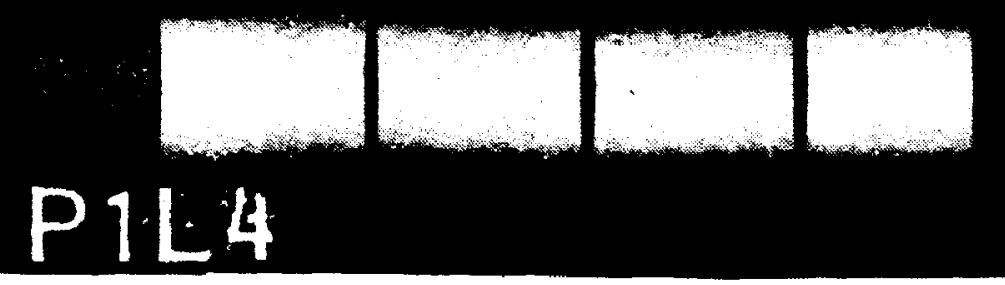

Fig. 8a. Pelletized fuel as fabricated

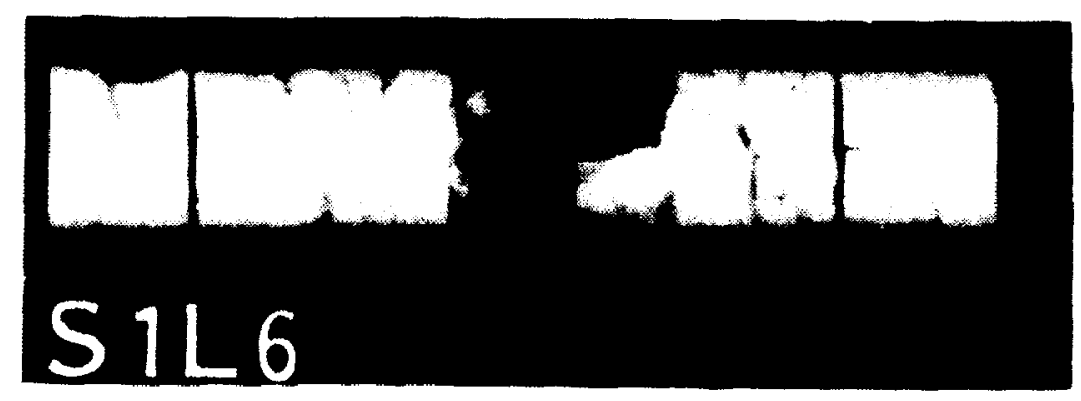

Fig. 8b. Random cracks in pellets

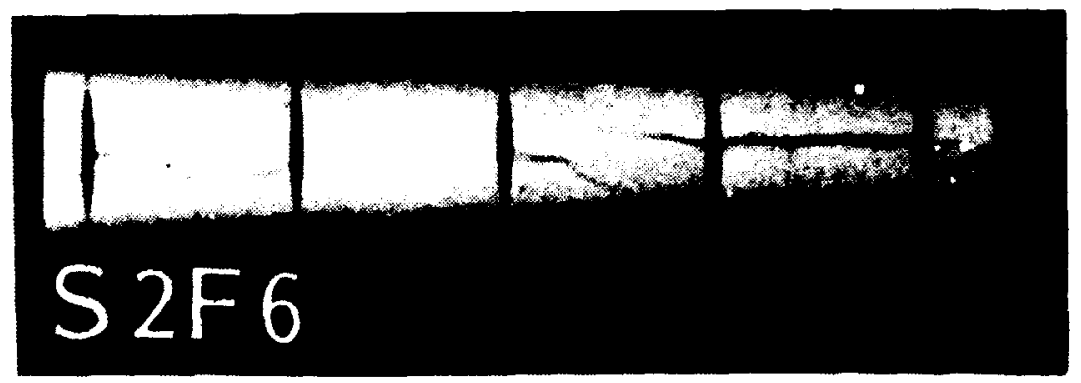

Fig. 8c. Longitudinal cracks in pellets

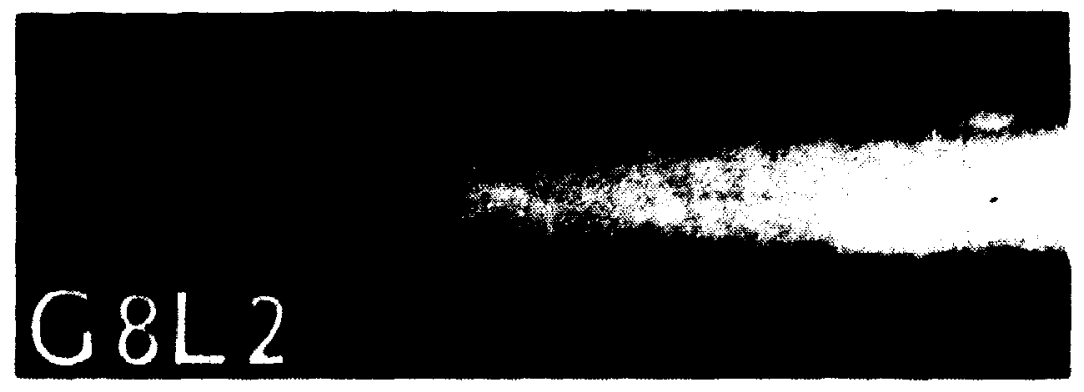

Fig. 8d. Central void in one pellet 


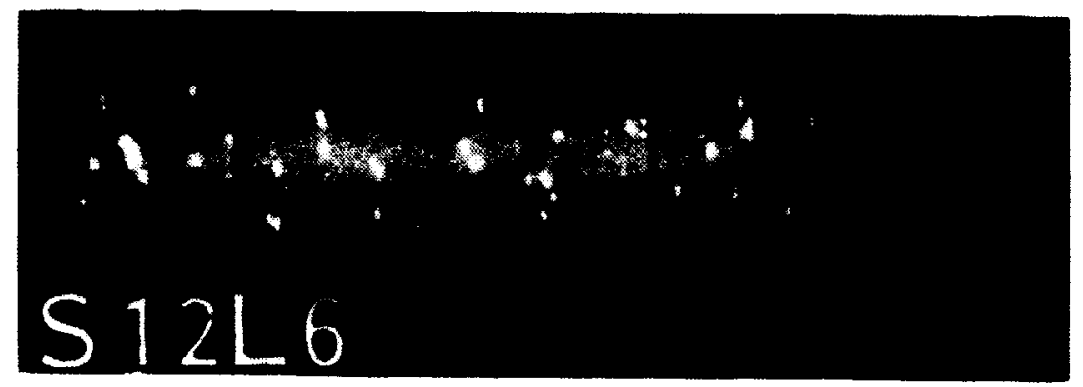

Fig. 8e. Inclusions of $\mathrm{Pu}$ in pellets

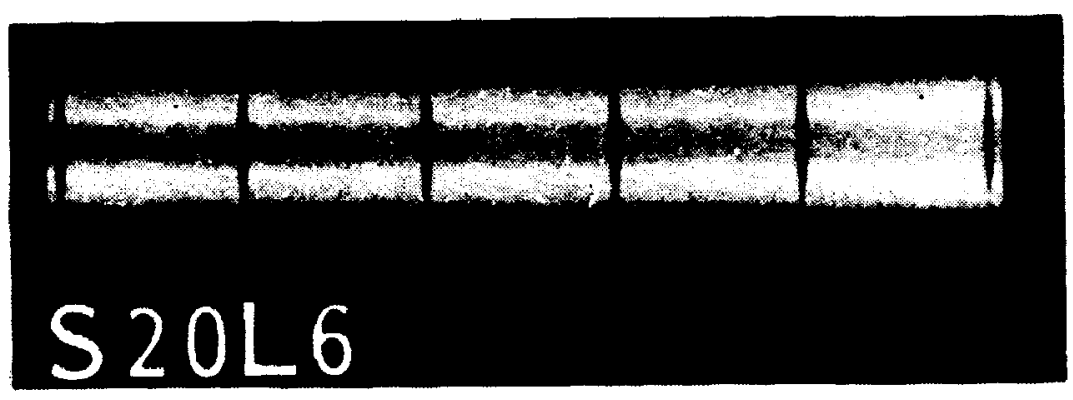

Fig. 9a. Annual fuel as fabricated

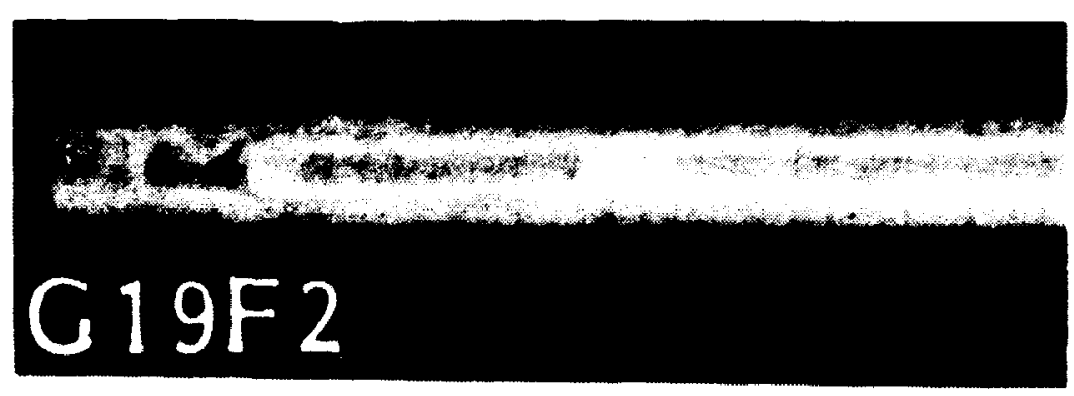

Pig. 9b. Accumulation of Pu in central vold

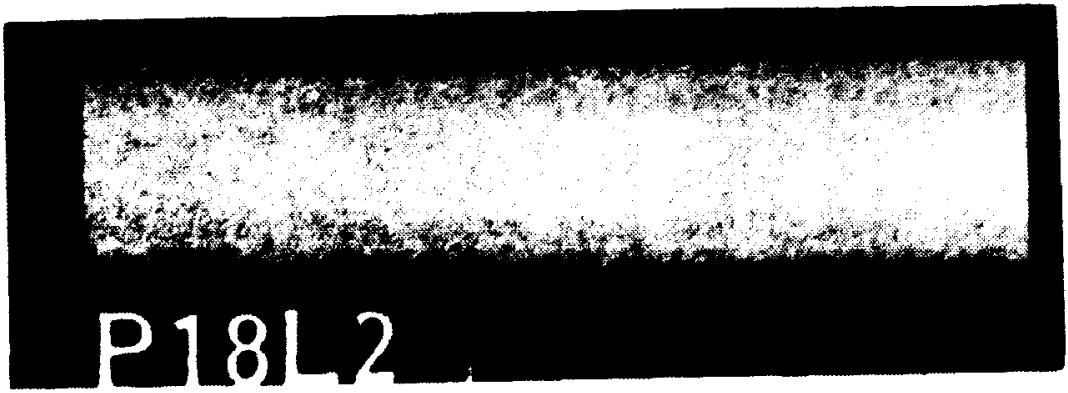

Pig. 10a. Vibro-compacted fuel as fabricated 


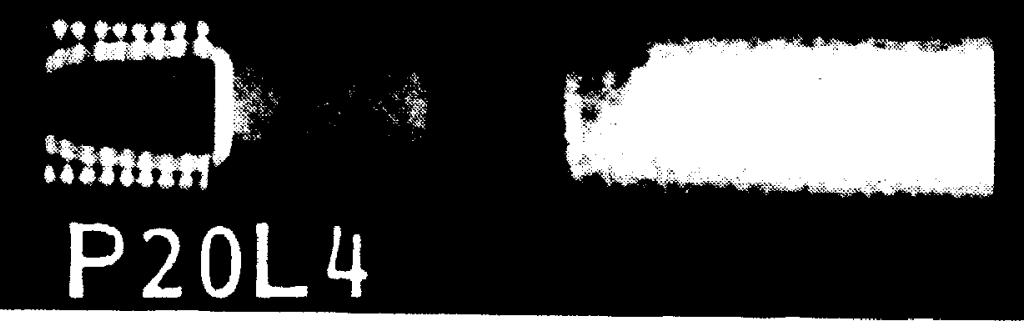

Fig. 10b. Missing chips in vibru-compacted fuel

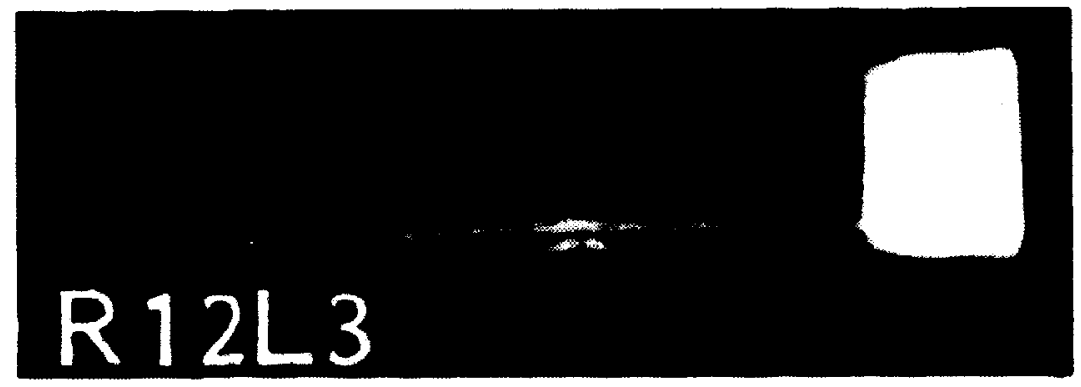

Fig. 11a. Deformed cladding

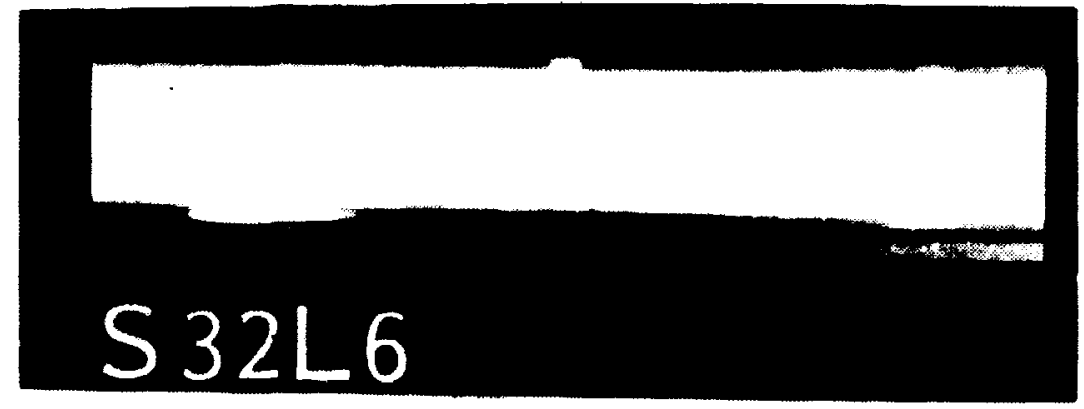

PIg. 11b. Hyarides in cladding

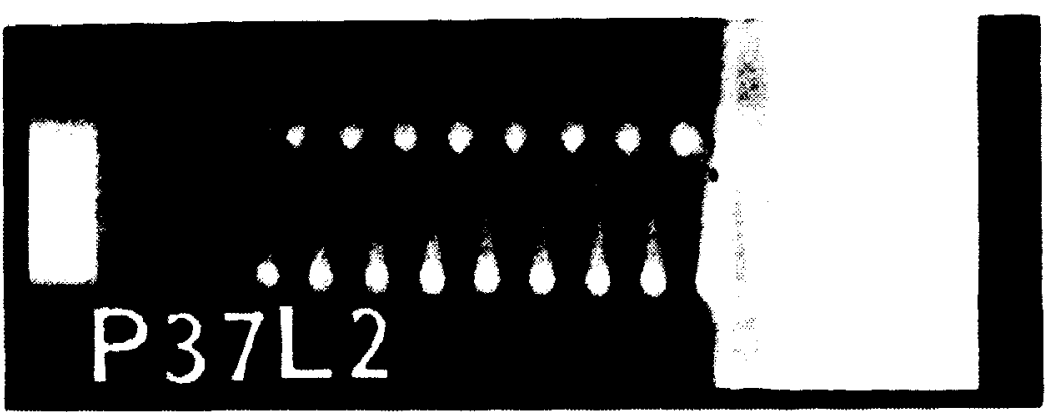

Fig. 12a, Plenum. Spring as fabricated 


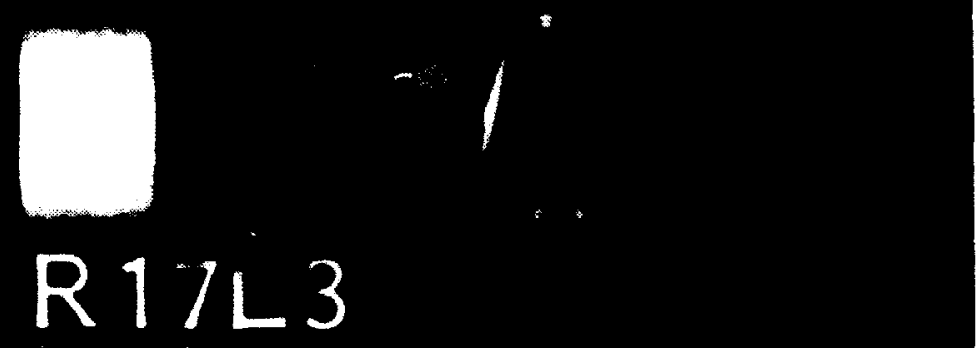

Fig. 12b. Plenum. Dislocated insulating disc

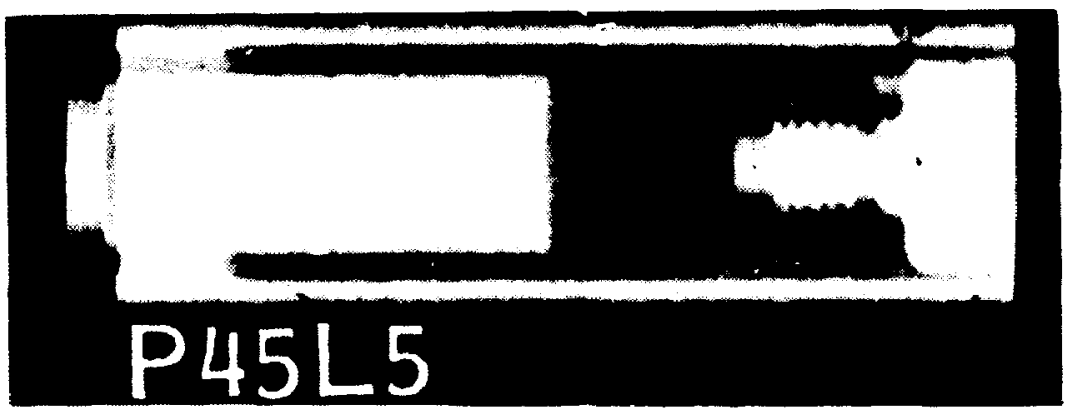

Fig. 13. Bottom plug as fabricated

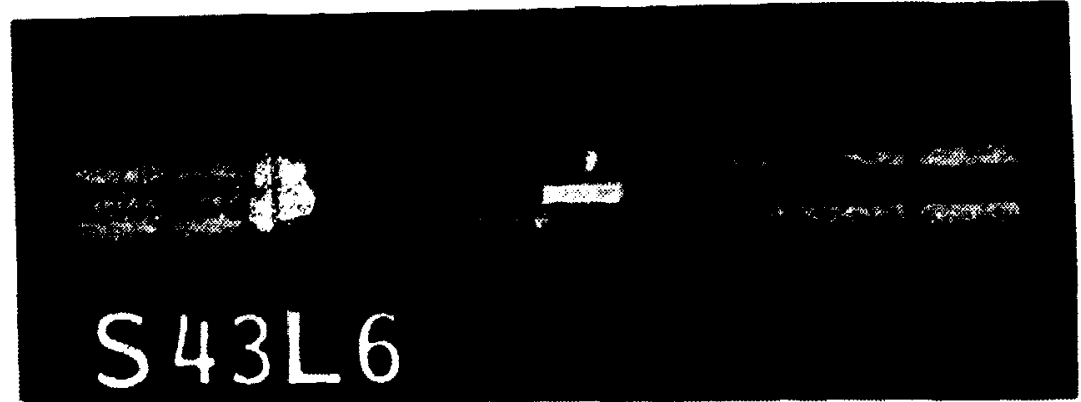

Pig. 14a.Melted thermocouple

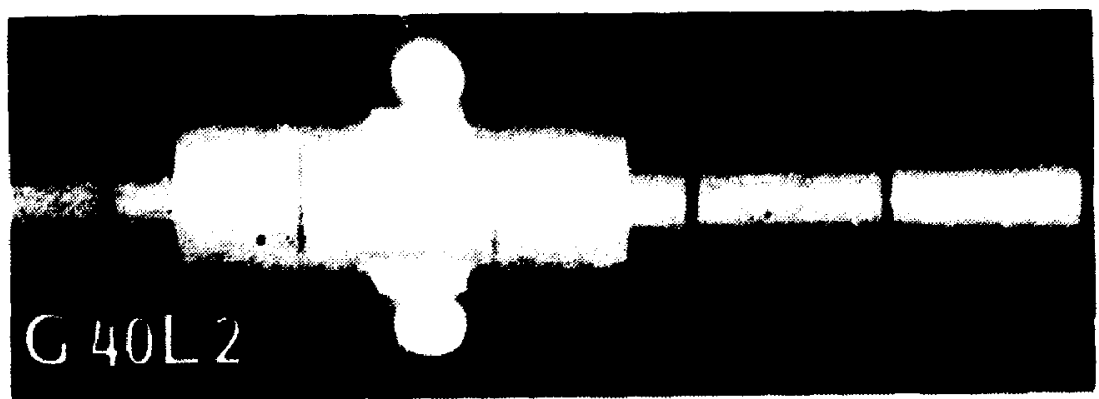

Fig. 14b. Diameter gauge as fabricated 
Hany more examples of the application of NR to the control of nuclear fuel can be found in [8] where a whole section was devoted to that problem (pp. 181 to 237).

An extensive review of the above problem is also given in [10] (pp. 221 to 247).

Part 5 of [3] (pp. 347 to 443) is also devoted to the applications of NR to nuclear fuel (13 papers on that subject).

Part 6 of [3] (pp. 445 to 530) is devoted to general applications of NR in the nuclear industry (10 papers). In this group of applications NR of reactor control assemblies is worth mentioning (described in [3] on pp. 447 to 459). Here an antimony-beryllium neutron source (pp. 453 to 459 and 367 to 374 in [3]) as well as californium-252 radioisotopic source (pp. 359 to 366 in [4]) was used.

\subsection{Industrial applications}

One of the most important application of NR is the control of explosives and pyrotechnical devices. Here NR can detect the presence or absence of explosive in a metallic device, a break in the explosive train, density changes, uniformity of explosives and inclusions of foreign materials. Whereas an $x$-ray radiograph can show the metallic parts of those devices, the NR radiograph shows the low atomic number materials (explosives, plastic, adhesives). The two ( $\mathrm{X}$ - and neutron radiographs) give supplementary findings.

Many interesting applications are described in a chapter on "Explosives and ordnance applications" (pp. 115 to 133) in [8], in [7] (pp. 18 to 20) and in [3] (pp. 77 to 82).

IR provides an excellent method for the detection of residual core material in cast turbine blades. The blades (mainly made of a nickel alloy) are checked by NR to be sure that no re-idual ceramic core material is left in the internal cooling passages of the blades. For that purpose 1 to 28 of gadolinia 
is added to the ceramic material, which thus attenuates neutrons more than the relatively more transparent nickel alloy. Here again reference can be made to a paper in [8] (pp. 152 to 157) as well as to a paper in [3] (pp. 77 to 82) and [4] (pp. 329 to 336 ).

NR is also widely applied in the aerospace industry, as described e.g. in "Aerospace applications" in [8] (pp. 135 to 180). NR played an important role in the Apollo missions, as described in [3] (pp. 325 to 332) as well as in the control of explosive chains of the Ariane launcher, described in [4] (pp. 263 to 270).

A general survey of industrial applications of NR in USA was given in [10] (pp. 291 to 326) as well as in [4] (pp. 47 to 54).

A short description of various industrial applications of NR is also given in (1] (pp. 62 to 64).

Low intensity, mobile neutron radiographic systems are used to control corrosion in aircraft. As neutron source a small accelerator (as described in [4] on pp. 407 to 414 and 447 to 454 ) or a californium-252 radioisotopic source (as described in [4] on pp. 431 to 438 ) is used.

Other NDT methods (ultrasonics, X-radiography, eddy-currents) are also used for the detection of corrosion. The NR methods of detection differ from the other NDT methods in that the primary detecting mechanism does not involve the change in the thickness of the basic material, but rather the accumulation of the corrosion product which contains hydrogen.

An investigation about the possibility of using real-time NR (see pp. 565 to 570 in [4]) has shown that the thickness of corrosion product that can be detected by that method is in the order of $70 \mu \mathrm{m}$ (corrosion product bulld-up in aluminium resulting from a metal loss as small as $25 \mu \mathrm{m}$ ). 
Neutron radiography combined with low-voltage $x$-radiography is used for inspecting of comcosite and composite-metal structures. This was reported in [7] (pp. 24 to 29) for boron fibre composites as well as in [4] (pp. 315 to 323) where NR has provided imaging of resin porosity and $x$-radiography showing the fiber distribution.

There are many other industrial applications of NR. They were reviewed in the chapter "other applications" in [8] and especially in the paper on pp. 252 to 267 describing such applications as rocket nozzle inspection, failure analysis (e.g. lack of detonators or o-rings, parachute harness, electrical relays), explosives.

Part 4 of [3] is devoted to those other industrial applications. It contains 8 papers on different subjects. In this category of applications the NR control of concrete is worth mentioning. In [4] (PF. 255 to 262) a method of studying the water-permeability of concrete is described, whereas other paper in [4] (pp. 321 to 328) illustrates the application of NR to the study of the internal structure and microcracking of concrete.

WR has also been used to the study of soil and rock. This was reported in [8] (pp. 241 to 251), where the use of californium252 for scanning inhomogeneities in moisture and density is described.

In a paper in [4] (pp. 271 to 279) the investigation by NR of mass transfer in a partially frozen soil is described. Here neutrons from a research reactor were used for the study.

The internal structure of electric contacts can be studied by NR, as described in [7] (Pp. 34 to 37). This method consists of applying a neutron absorbing grease (e.g. grease rich in hydrogen, borated grease or one containing gadolinium sulphate) to the surface of the contacts prior to their closing. After closing the contacts the grease will be forced to leave the surfaces of good contact and concentrate in the zones of bad con- 
tact. Those zones will be then detected by NR.

Electronic devices such as relays are inspected by NR to detect foreign materials that might interfere with their operation.

Hechanical connectors and assemblies can be usefully studied by NR. The presence or absence or the displacement of sealing orings can be revealed by NR (see e.g. pp. 281 to 393 in [4]).

Also noneycomb assemblies for aerospace or other applications can be inspected by NR to show adhesives during manufacture or repair, or to show the presence of water during service.

Two phase counter flow in heat pipes was studied by NR and is described in [4] (pp. 609 to 616).

NR finds many applications in metalluray. This was described in detail in [10] (pp. 327 to 359). With NR one can observe the distribution of high neutron cross section alloying agents (such as cadmium), hydriding of materials (such as zirconium or titanium), distribution of other agents (such as boron or 1ithium).

The real-time NR (or neutron fluoroscopy) is especially worth mentioning as a special tool for industrial applications of NR. That use was discussed in many papers during the first [3] as well as the second [4] World Conference on NR. (See part 8 in [3] containing 8 papers on pp. 599 to 669 and part VIII in [4] with 13 papers on pp. 525 to 632 ).

The most spectacular application of neutron fluoroscopy is no doubt in the study of engine fluids in aero engines and automative engines. This was described in [3] in two papers (pp. 625 to 642) as well as in [4] in the paper on pp. 579 to 586.

The equipment itself used for NR fluoroscopy is described in [4] (pp. 527 to 535 and 595 to 600).

Neutron fluoroscopy was used also in other applications of NR 
(as e.g. for corrosion detection described in [4], pp. 563 to 570).

\subsection{Non-industrial applications}

Although the non-industrial applications of NR are not so numerous as the industrial ones, many are worth mentioning here.

The papers presented on those subject at [3] were grouped in part 7 (pp. 531 to 598 containing 7 papers) and in part VII of [4] (pp. 471 to 524 containing 6 papers).

Most of those applications relate to biology, medicine and dentistry.

The application of NR to histopathology was described in [8] (pp. 77 to 86). This subject was further treated in [3] (pp. 533 to 553).

The problem of determining the presence of intraosseus tumor tissues by NR (described in [8], pp. 77 to 86) was further discussed in [4] (pp. 513 to 518) where it was also demonstrated how NR can examine nondestructively the interface between metal implants and the host mandible.

These problems relate directly to dentistry. The applications of NR in that field were described in [3] (pp. 555 to 563).

A more general study of the applications of NR in biology and medicine were given in [3] (pp. 573 to 580) where e.g. changes in the lumbar spine were detected by NR. This example was further demonstrated in [4] (pp. 639 to 646).

Concluding the review of non-industrial applications of NR one can mention two further interesting applications. The $s 3 a t$ is the study of young roots growing in soll ([3], Pp. $5 \approx 2$ te 590) and the second the forensic utilization of NR ([3], Nn. 591 to 598). 
In the group of special applications of NR ([4], pp. 471 to 524, 6 papers) the use of NR in the investigation of art objects (metal objects and paintings) is described.

In [4] (pp. 489 to 496) examples are given of the use of NR for examination of excavated ancient relics (as e.g. bronze and iron objects).

The oil paintings of Rembrandt and other artists were investigated by a neutron autoradiography method. Although this cannot be described strictly as ncutron radiography, it is worth mentioning here, as it gives very interesting and valuable results. This method, described in [4] (pp. 519 to 524) and in $[13,14]$ consists of activating the painting under investigation by neutrons coming from a reactor. Thereafter a contact autoradiography of the radioactive painting is made on an $x$-ray film and at different spots of the painting (containing different radioisotopes) a spectral analysis of the emitted gama radiation is made. Also half-lives of the particular radioisotopes are measured. The autoradiographs make visible underlying structures like prepal tory sketches, conceptional changes, signatures and the individual characteristics of the artist's brush-work to enable the work to be certified as genuine.

The technique described has the advantage of not being confined to canvas paintings only.

Using the neutron autoradiographic technique it was ascertained that the world-renowned painting "The man with the golden helmet" attributed to Rembrandt was in fact not pairited by him.

\section{ACKNOWLEDGEMENTS}

Several authors of papers published in the proceedings of the II WCNR [4] have supplied sides illustrating their different applications of neutron radiography. Those slides will be used during the oral presentation of this paper. The contribution of the following authors is gratefully acknowledged herewith: P.A. Attwood (Shell Research Ltd.), E. Dühmke (Georg-August Univer- 
sität, Göttingen), D.H.C. Harris (UKAEA Harwell), G.-I. Mazsumoto (Nagoya University), P.E. Underhill (Aerotest Operations Inc.), W.L. Whittemore (GA Technologies Inc.).

\section{REFERENCES}

1. P. von der hardt, H. Röttger (editors) . Neutron radiography handbook. D. Reidel Publishing Company, 1981, EUR 7622e, ISBN 90-227-1378-2.

2. J.C. Domanus (editor). Reference neutron radiographs of nuclear reactor fuel. D. Reider Publishing Co., 1984. EUR 8916 EN EP ISBN 90-277-1717-6.

3. J.P. Baron, P. von der Hardt (editors). Neutron radiography. Proceedings of the First World Conference, San Diego, California, U.S.A., 7-10.12.1981.

4. J.P. Barton, G. Farny, J.-L. Person, H. Röttger (editors). Neutron radiography. Proceedings of the second World conference, Paris, France, 16-20.06.1986).

5. J.C. Domanus. Collimators for thermal neutron radiography. An overview. D. Reidel Publishing Co., 1987. J.F.W. Markgraf, editor.

6. H. Berger. Neutron radiography. Methods, capabilities, and applications. Elsevier Publishing Co., Amsterdam/London/ New York. 1965.

7. La neutronographie. Kodak-Pathe. 1974.

8. H. Berger (editor). Practical applications of neutron radiography and gauging. ASTM STP 586. 1976.

9. J.P. Barton. Neutron radiography 1964-1977. The American society for Nondestructive Testing. Columbus, Ohio, USA 1977. 
$-30-$

10. Atomic Energy Review, Vol. 15, No 2, June 1977.

11. L.E. Bryant (technical editor), P. McIntire (editor). Nondestructive testing handbook. Second Ed., Vol. 3. Radiography and radiation testing. Anerican Society for Mondestructive Testing. 1985.

12. H. Berger, D.C. Cutforth, D.A. Garrett, J. Haskins, F. Iddings, R.L. Newacheck. Neutron radiography. Section 12 in [11].

13. J. Kelch (editor). Der Mann mit den Goldhelm. Genäldegalerie Staatiiche Museen Preussischer Kulturbesitz. BerIin 1986.

14. C.0. Fischer, J. Kelch, C. Laureuze, K. Slusallek. Autoradiography of paintings after neutron activation at a cold neution guide. Atomkernenergie. Kerntechnik, 51, (1987) No. 1, 9-13. 
NEUTRON RADIOGRAPHY

Techniques and applications

J.C. Domanus

\begin{tabular}{|c|c|c|c|c|c|c|}
\hline reves 32 & Tables & Imsistrations & 14 & Roferences & 14 & $87-550-1371-6$ \\
\hline
\end{tabular}

Abstract. After describing the principles of the "in pool" and "dry" installations, techniques used in neutron radiography are reviewed. Use of converter foils with silver halide films for the direct and transfer methods is described. Advantages of the use of nitrocellulose film for radiographying radioactive objects are discussed. Dynamic imaging is shortly reviewed. Standardization in the field of neutron radiography (ASTM and Euratom Neutron Radiography Working Group) is described. The paper reviews main fields of use of neutron radiography. Possibilities of use of ncutron radiography at research reactors in various scientific, industrial and other fields are mentioned. Examples are given of application of neutron radiography in industry and the nuclear field.

This paper will be iresented at the Seminar "Procedes de Control Non Destructifs" in Liége, Belgium ( 4 \& February 1988) and at the Uanish-Israeli Symposium on Non-Destructive Evaluation in Lyngby, Denmark (27 June - 1 July 1988).

Decriptors - INIS

IMAGE PROCESSING; NEUTRON RADIOGRAPHY; NITROCELLULOSE; NONDESTRUCTIVE TESTING; PHOTOGRAPHIC FILMS; STANDARDIZATION; USES 
Available on request from. Rise Library,

Riso National Laboratory, P.O. Box 49,

DK-4000 Roskilde, Denmark

ISBN 87-550-1371-6

Phone (02) 371212 ext.2262

ISSN 0418-6435 